\title{
MEASURABLE HILBERT SHEAVES
}

\author{
MICHAEL A. WENDT
}

(Received 14 March 1994; revised 6 February 1995)

Communicated by R. H. Street

\begin{abstract}
We describe measurable Hilbert sheaves as Hilbert space objects in a sheaf category constructed from a measure space. These are quite useful for the interpretation of the direct integral of Hilbert spaces as an indexed functor. We set up a framework to put this and similar constructions of operator theory on an indexed categorical footing.
\end{abstract}

1991 Mathematics subject classification (Amer. Math. Soc.): primary 18B25, 18D35; secondary 28A50, 47B40.

\section{Introduction}

The direct integral of Hilbert spaces, $\int^{\oplus} \mathscr{H}(x) d \mu(x)$, exhibits both a measureindexed nature and a coproduct-like nature. The question arises: can a suitable universal property be found for it? For example, is it a measure-indexed coproduct? The answer seems to be no. More precisely, reasonable (from an operator theoretic point of view) categories of measure spaces do not have products. Constant families (that is, $\Delta$ of [4]) would then be problematic.

However, we can make sense of 'measurable Hilbert families' and interpret the direct integral as an indexed functor to set up a systematic, categorical framework for this and similar constructions. Our project is to describe the elements of the diagram:

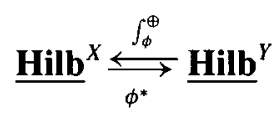

for $X$ and $Y$ measure spaces and $\phi$ some appropriate morphism of such. This amounts to understanding $X$-indexed families of Hilbert spaces and substitution. We may

(C) 1996 Australian Mathematical Society $0263-6115 / 96 \$ A 2.00+0.00$ 
'approximate' classical indexed category theory well in this context by constructing a categorization and generalization of the direct integral. In this paper, we put forth an approximation where families are Hilbert space objects in a sheaf topos constructed from the measure space $X$ : $\underline{\text { Hilb }}^{X}=\underline{\mathbf{H i l b}}(\operatorname{Sh}(X))$. The construction 'Sh $(X)$ ' embeds measure spaces in topoi.

We wish to use actual measure spaces for the base. The plan is to determine how far classical measure theory can go in an indexed category setting. However, there are other possibilities for a base category. One might consider Grothendieck topoi as an appropriate base since these have finite products (and $\Delta$ ). A similar possibility is to 'close up' the image of a measure space category under products in the category of topoi. These ideas will await future work.

One important aspect of our work here is the introduction of a suitable notion of measurable Hilbert sheaf (defined in Section 4.3). This will be a Hilbert space object in the topos. In particular, in Section 4.2, we describe how to construct a sheaf from the classical notion of a measurable field of Hilbert spaces. It is our motivating example of a Hilbert sheaf and is of interest to analysts.

The second major aspect of this work is to interpret the direct integral in the indexed categorical setting. For a Hilbert sheaf, $H$, we define $\int^{\oplus} H$ as the set of 'square-integrable' global sections of $H$. With a suitable norm, this is a Hilbert space. The construction is functorial.

A certain special structure, called a disintegration, on a morphism of measure spaces, $\phi$, is enough to define a relative direct integral, $\int_{\phi}^{\oplus} H$, as square-integrable sections on the fibres of $\phi$ (the essence of a disintegration is that the fibres of $\phi$ are given measures, so that measurement in the domain is obtained by integrating fibrewise measurements over the codomain). This generalizes $\int^{\oplus} H$ and is also functorial. Moreover, $\int_{-}^{\oplus}$ is pseudo-functorial with domain measure spaces and disintegrations.

We conclude by discussing connections with indexed category theory. The elements of the above diagram become:

(1) $\underline{\text { Hilb }}^{X}=\underline{\text { Hilb }}(\operatorname{Sh}(X))$,

(2) $\phi^{*}$ is the lifting, via an appropriate notion of Cauchy completion, of change of base $\operatorname{Sh}(X) \stackrel{\phi^{*}}{\longrightarrow} \operatorname{Sh}(Y)$ to Hilbert sheaves, and $\int_{\phi}^{\oplus}$ as the relative direct integral.

The author would like to thank the referee for helpful and constructive comments.

\section{Categories of measure spaces}

Notation. Measurable spaces are denoted by pairs, $(X, \mathscr{A}),(Y, \mathscr{B})$, etcetera, consisting of a set and a $\sigma$-algebra. Mble denotes the category of measurable spaces 
and measurable functions. Measure spaces will be denoted by triples, $(X, \mathscr{A}, \mu)$, $(Y, \mathscr{B}, v)$, etcetera, consisting of a measurable space and a measure.

We will assume that singletons are measurable and measure spaces have finite measure. These are usually called 'finite measure spaces'. Two categories of measure spaces will be considered:

DEFINITION 2.1. A measurable function, $(X, \mathscr{A}, \mu) \stackrel{f}{\longrightarrow}(Y, \mathscr{B}, v)$ is called measure zero reflecting or simply $M O R$ if $\nu(B)=0 \Rightarrow \mu\left(f^{-1}(B)\right)=0$. M0R is the category whose objects are finite measure spaces and whose morphisms are measure zero reflecting.

DEFINITION 2.2. An object of Disint is a finite measure space. A morphism, called a disintegration, between two objects, $(X, \mathscr{A}, \mu)$ and $(Y, \mathscr{B}, \nu)$, consists of an $(X, \mathscr{A}) \stackrel{f}{\longrightarrow}(Y, \mathscr{B}) \in \underline{\text { Mble }}$ and a family $\left(X_{y}, \mathscr{A}_{y}, \mu_{y}\right)_{y \in Y}$ of finite measure spaces, where $X_{y}:=f^{-1}(y)$ and $\mathscr{A}_{y}=\left\{A \cap f^{-1}(y) \mid A \in \mathscr{A}\right\}$ subject to two axioms:

(1) $\forall A \in \mathscr{A}$, the map $y \mapsto \mu_{y}\left(A \cap f^{-1}(y)\right)$ is measurable and bounded and

(2) $\forall A \in \mathscr{A}, \mu(A)=\int_{Y} \mu_{y}\left(A \cap f^{-1}(y)\right) d v(y)$.

A disintegration is denoted by $(X, \mathscr{A}, \mu) \stackrel{\left(f, \mu_{y}\right)}{\longrightarrow}(Y, \mathscr{B}, \nu)$. These form a category with identity as $(X, \mathscr{A}, \mu) \stackrel{\left(1_{x}, t_{x}\right)}{\longrightarrow}(X, \mathscr{A}, \mu)$ where $1_{X}$ is the identity function and $\iota_{x}$ is counting measure on $\mathscr{I}_{x}=\left\{A \cap 1^{-1}(x) \mid A \in \mathscr{A}\right\}$, the discrete $\sigma$-algebra on $\{x\}$. For $(X, \mathscr{A}, \mu) \stackrel{\left(f, \mu_{y}\right)}{\longrightarrow}(Y, \mathscr{B}, \nu) \stackrel{\left(g, \nu_{z}\right)}{\longrightarrow}(Z, \mathscr{C}, \rho)$, the composite is $(X, \mathscr{A}, \mu) \stackrel{\left(g f, \theta_{z}\right)}{\longrightarrow}$ $(Z, \mathscr{C}, \rho)$ where

$\theta_{z}(E):=\int_{g^{-1}(z)} \mu_{y}\left(E \cap f^{-1}(y)\right) d \nu_{z}(y) \quad$ for $\quad E \in \mathscr{E}_{z}=\left\{A \cap f^{-1} g^{-1}(z) \mid A \in \mathscr{A}\right\}$.

For an extensive list of examples and basic properties, see [7]. Some useful results are:

\section{PROPOSITION 2.1. (i) MOR and Disint have}

(a) an initial object given by $(\emptyset,\{\emptyset\}, 0)$,

(b) a terminal object given by $(1,2$, counting),

(c) binary coproducts given by $(X, \mathscr{A}, \mu)+(Y, \mathscr{B}, \nu)=(X+Y, \mathscr{A}+\mathscr{B}, \mu+$ v) (the $\sigma$-algebra consists of sets of the form $A+B$ and $(\mu+\nu)(A+B)=$ $\mu(A)+v(B))$, and

(d) these coproducts are disjoint.

(ii) M0R and Disint are monoidal categories. The unit is given by (b) above and the $\otimes$ is the usual product of measure spaces (the $\sigma$-algebra is generated by measurable rectangles; we do not assume it is complete). 
(iii) $\left(f, \mu_{y}\right) \in \underline{\text { Disint }} \Rightarrow f \in \underline{\mathbf{M O R}}$.

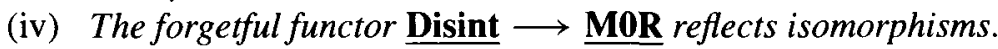

For future reference (Propositions 5.8 and 5.10), we state an important property of disintegrations proved in [7]:

PROPOSITION 2.2. Let $(X, \mathscr{A}, \mu) \stackrel{\left(f, \mu_{y}\right)}{\longrightarrow}(Y, \mathscr{B}, v)$ be a disintegration. For $X \stackrel{a}{\rightarrow} \mathbf{R}$ an integrable function,

$$
\int_{X} a(x) d \mu(x)=\int_{Y} \int_{f^{-1}(y)} a(x) d \mu_{y}(x) d \nu(y) .
$$

We next show that $\underline{\mathbf{M} 0 \mathbf{R}}$ does not have products, which is pertinent to the development of indexed category theory over Disint.

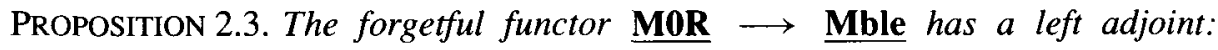
$(X, \mathscr{A}) \mapsto(X, \mathscr{A}, 0)$ where $0(A)=0$ for all $A \in \mathscr{A}$.

ProOf. Any measurable function out of $(X, \mathscr{A}, 0)$ is MOR.

COROLLARY. MOR does not have products.

PROOF. We exhibit a contradiction for a particular example. Let $((0,1), \mathscr{L}, \lambda)$ be the Lebesgue open unit interval. Assume $((0,1) \times(0,1), \mathscr{L} \otimes \mathscr{L}, \rho)$ is the product in M0R (by the proposition, the underlying measurable space must be the product in Mble).

For each $t \in[1, \infty)$, the function $(0,1) \stackrel{f_{t}}{\longrightarrow}(0,1) ; x \mapsto x^{t}$ is M0R. Then $(0,1) \stackrel{\left(i, f_{t}\right)}{\longrightarrow}(0,1) \times(0,1)$, where $i$ denotes the inclusion, is M0R. Thus, for each $t, \rho$ (Image $\left.\left(f_{t}\right)\right)$ must be non-null since $\lambda(0,1)$ is; $t$ ranging over $[1, \infty)$ provides a continuum of disjoint, non- $\rho$-null sets, in which case, $\rho((0,1) \times(0,1))=\infty$ contradicting finiteness of measure.

\section{Sheaves on a measure space}

3.1. Definition Let $(X, \mathscr{A}, \mu)$ be a measure space. $S h(X)$ denotes the sheaf category whose objects we call measurable sheaves (see also [2, p. 25]). The site has the poset $(\mathscr{A}, \subseteq)$ as underlying category and a countable family $\left\{A \supseteq A_{n} \in \mathscr{A}\right\}_{n=1}^{\infty}$ will be a cover of $A \in \mathscr{A}$ if $\mu\left(A \backslash \bigcup_{n=1}^{\infty} A_{n}\right)=0$.

Notation. For a presheaf $F, \rho_{A^{\prime}}^{A}: F(A) \longrightarrow F\left(A^{\prime}\right)$ denotes restriction to $A^{\prime} \subseteq A$. 
In general, representables are not sheaves, for consider:

(Counter)Example 1. Let $A, A^{\prime} \in \mathscr{A}, A \subseteq A^{\prime}, A \neq A^{\prime}$, and $\mu\left(A^{\prime} \backslash A\right)=0$. Then $\mathscr{A}\left(A^{\prime}, A\right)=\emptyset$ and $\mathscr{A}(A, A)=1$. $A^{\prime}$ covers $A$, so if $\mathscr{A}(-, A)$ were a sheaf, we would have $\mathscr{A}\left(A^{\prime}, A\right)=\mathscr{A}(A, A)$.

The associated sheaf of $\mathscr{A}(-, A)$ is

$$
a(\mathscr{A}(-, A))\left(A^{\prime}\right)= \begin{cases}1 & \mu\left(A^{\prime} \backslash A\right)=0, \\ \emptyset & \text { else. }\end{cases}
$$

An alternate sheaf category is suggested by ' $\mu\left(A^{\prime} \backslash A\right)=0$ '.

DEFINITION 3.1. Let $\mathscr{N}$ be the ideal of null sets in $\mathscr{A}$. Then $\mathscr{A} / \mathscr{N}$ is a category with an arrow $\bar{A} \longrightarrow \bar{B}$ if and only if there are two representatives, $A_{0}$ of $\bar{A}$ and $B_{0}$ of $\bar{B}$, such that $A_{0} \subseteq B_{0}$. Given $\bar{A} \longrightarrow \bar{B} \longrightarrow \bar{C}$, with $A_{0} \subseteq B_{0}$ and $B_{1} \subseteq C_{1}$, we have a composite $\bar{A} \longrightarrow \bar{C}$ via $A_{0} \cap B_{1} \subseteq B_{0} \cap B_{1} \subseteq C_{1}$. We say $\left\{\overline{A_{n}}\right\}_{n=1}^{\infty}$ is a cover of $\bar{A}$ if $\bigcup_{n} \overline{A_{n}}=\bar{A}$ (we may define $\bigcup_{n} \overline{A_{n}}=\overline{\bigcup_{n} A_{0 n}}$ where $A_{0 n}$ is any choice of representatives). $S h(\mathscr{A} / \mathscr{N})$ denotes the category of sheaves for this site.

PROPOSITION 3.1. $\operatorname{Sh}(X) \simeq \operatorname{Sh}(\mathscr{A} / \mathscr{N})$

PROOF. Use the axiom of choice to pick a particular representative $r(\bar{A})$ of each equivalence class $\bar{A} \in \mathscr{A} / \mathscr{N}$. The equivalence

$$
\operatorname{Sh}(X) \underset{()_{*}}{\stackrel{()^{*}}{\leftrightarrows}} \operatorname{Sh}(\mathscr{A} / \mathscr{N})
$$

is given as follows: for $F \in S h(X), F_{\star}(\bar{A})=F(r(\bar{A}))$ and for $G \in S h(\mathscr{A} / \mathscr{N})$, $G^{\star}(A)=G(\bar{A})$.

Representables become sheaves after passing to $\operatorname{Sh}(\mathscr{A} / \mathscr{N})$. Indeed, we have:

PROPOSITION 3.2. Definition 3.1 provides the canonical topology on $\mathscr{A} / \mathscr{N}$.

PROOF. It is straightforward to check that representables are sheaves for this topology. Suppose $\mathscr{K}$ is another topology for which representables are sheaves. We must show, for a $\mathscr{K}$-cover $\left\langle\overline{A_{t}}\right\rangle$, there is a countable sub-family $\left\langle A_{t_{n}}\right\rangle$ such that $\bigcup \overline{A_{t_{n}}}=\bar{A}$. Let

$$
\alpha=\sup \left\{\mu\left(\bigcup A_{t_{n}}\right) \mid\left\langle\overline{A_{t_{n}}}\right\rangle \text { countable subsequence of }\left\langle\overline{A_{t}}\right\rangle\right\}
$$


The supremum exists since $\mu\left(\bigcup A_{t_{n}}\right) \leq \mu(A)<\infty$. For each $k$, let $\left.\overline{\left\langle A_{t_{n}}\right.}\right\rangle$ be a sequence with $\mu\left(\bigcup_{n} A_{t_{n k}}\right) \leq \alpha-1 / k$ and let $A_{t_{n}}=\bigcup_{k=1}^{\infty} A_{t_{n k}}$. Then $\mu\left(\bigcup A_{t_{n}}\right)=\alpha$ and $\bar{B}:=\overline{\bigcup A_{t_{n}}}=\bigvee \overline{A_{t}}$ (supremum taken in $\mathscr{A} / \mathscr{N}$ ) by maximality of $\alpha$.

Now, $[-, \bar{B}]$ is a $\mathscr{K}$-sheaf. In the equalizer

$$
[\bar{A}, \bar{B}] \longrightarrow \prod_{t}\left[\overline{A_{t}}, \bar{B}\right] \Longrightarrow \prod_{s, t}\left[\overline{A_{s}} \cap \overline{A_{t}}, \bar{B}\right]
$$

$\left[\overline{A_{t}} \cap \overline{A_{t}}\right]$ and $\left[\overline{A_{t}}, \bar{B}\right]$ are equal to 1 for each $s$ and $t\left(\bar{B}=\bigvee \overline{A_{t}}\right.$ so $\left.\overline{A_{t}} \leq \bar{B}\right)$ so both products are 1 in which case $[\bar{A}, \bar{B}]=1$. This implies $\bar{A} \leq \bar{B}$. But, $\bar{B} \leq \bar{A}$ since $\bar{B}=\bigvee \overline{A_{t}}$ and $\overline{A_{t}} \leq \bar{A}$. Thus, $\bar{A}=\bar{B}=\overline{\bigcup A_{t_{n}}}$ as required.

COROLLARY. Sh(X) satisfies the axiom of choice.

PROOF. $\mathscr{A} / \mathscr{N}$ is a complete Boolean algebra and sheaves on such is a topos with the axiom of choice (see, for example, [5, p.215]).

REMARKS. (1) We will implicitly assume that statements made in a measure theoretical context are 'up to almost everywhere equivalence.' Such a caveat is avoided in $\operatorname{Sh}(\mathscr{A} / \mathscr{N})$ where the 'modding out' is done once and for all at the beginning. We use $S h(\mathscr{A} / \mathscr{N})$ and $S h(X)$ interchangeably but the latter is more appropriate for our indexed category theory setting. When the reader sees $\bar{A}$, the context is $\operatorname{Sh}(\mathscr{A} / \mathscr{N})$. Otherwise, it is $\operatorname{Sh}(X)$.

(2) An example of the occurance of the caveat is the following: the corollary above suggests that our logic is essentially classical up to almost everywhere equivalence.

3.2. Examples and properties We now list some objects of $S h(X)$ and $S h(\mathscr{A} / \mathscr{N})$. We have already noted that:

EXAMPLE 1. $a(\mathscr{A}(-, A))\left(A^{\prime}\right)=\left\{\begin{array}{ll}1 & \mu\left(A^{\prime} \backslash A\right)=0 \\ \emptyset & \text { else }\end{array}\right.$ is a sheaf.

EXAMPLE 2. $1(A)=1, \forall A \in \mathscr{A}$. This is a terminal object of $\operatorname{Sh}(X)$.

EXAMPLE 3. $O(A)=\left\{\begin{array}{ll}1 & \mu(A)=0 \\ \emptyset & \text { else. }\end{array}\right.$ This is an initial object of $\operatorname{Sh}(X)$.

EXAMPLE 4. Let $(Y, \mathscr{B})$ be a measurable space. Define an object in $\operatorname{Sh}(\mathscr{A} / \mathscr{N})$ by $M_{Y}(-):=a(\underline{\operatorname{Mble}}(-, Y))$ (that is, $M_{Y}(\bar{A})$ is the associated sheaf of $\underline{\text { Mble }}(-, Y)$ evaluated at $\bar{A} \in \mathscr{A} / \mathscr{N}$ ). 
PROPOSITION 3.3.

$$
\begin{aligned}
M_{Y}(\bar{A})=\left\{\left(A_{0}, f\right) \mid\right. & A_{0} \in \mathscr{A}, A_{0} \subseteq A, \mu\left(A \backslash A_{0}\right)=0, \\
& \left.\left(A_{0},\left.\mathscr{A}\right|_{A_{0}}\right) \stackrel{f}{\longrightarrow}(Y, \mathscr{B}) \text { measurable }\right\} / \sim,
\end{aligned}
$$

with $\left(A_{0}, f\right) \sim\left(A_{0}^{\prime}, f^{\prime}\right)$ if and only if $\mu\left\{x \in A_{0} \cap A_{0}^{\prime} \mid f(x) \neq f^{\prime}(x)\right\}=0$.

Proof. Let $\left\{\overline{A_{n}}\right\}_{n=1}^{\infty}$ be a cover of $\bar{A}$ and let $\left(A_{0 n}, f_{n}\right)$ be the representatives of a compatible family in the $M_{Y}\left(\overline{A_{n}}\right)$ 's. Then

$$
\mu\left(A \backslash \bigcup_{n} A_{0 n}\right) \leq \mu\left(A \backslash \bigcup_{n} A_{n}\right)+\sum_{n} \mu\left(A_{n} \backslash A_{0 n}\right)=0 .
$$

Let $C_{n}=A_{0 n} \backslash \bigcup_{i<n} A_{0 i}$. The $C_{n}$ 's are pairwise disjoint and $\bigcup_{n} C_{n}=\bigcup_{n} A_{0 n}$. Define $f: \bigcup_{n} C_{n} \longrightarrow Y$ as follows: $x \in \bigcup_{n} C_{n}$ implies $x$ is in a unique $C_{n}$; put $f(x)=f_{n}(x)$. Then $\left.f\right|_{C_{n}}=\left.f_{n}\right|_{C_{n}}$ by construction and $f$ is measurable for if $B \in \mathscr{B}$, then $f^{-1}(B)=\bigcup_{n} f_{n}^{-1}(B) \cap C_{n} \in \mathscr{A}$.

We need only show that this definition of $f$ respects $\sim$. Suppose $\left(A_{0 n}, f_{n}\right) \sim$ $\left(A_{1 n}, g_{n}\right)$ for each $n$. Then $\left(C_{n},\left.f_{n}\right|_{C_{n}}\right) \sim\left(D_{n},\left.g_{n}\right|_{D_{n}}\right)$ where $D_{n}=A_{1 n} \backslash \bigcup_{i<n} A_{i 1}$, for $\mu\left\{x \in C_{n} \cap D_{n} \mid f_{n} \neq g_{n}\right\} \leq \mu\left\{x \in A_{0 n} \cap A_{1 n} \mid f_{n} \neq g_{n}\right\}=0$. We claim $\left(\bigcup_{n} C_{n}, f\right) \sim\left(\bigcup_{n} D_{n}, g\right)$. Let $x \in \bigcup_{n} C_{n} \cap \bigcup_{n} D_{n}$ and $f(x)=f_{n_{0}}(x), g(x)=$ $g_{n_{1}}(x)$. Then $f(x) \neq g(x)$ implies $f_{n_{1}}(x) \neq g_{n_{1}}(x)$ or $f_{n_{0}}(x) \neq f_{n_{1}}(x)$. Each of the latter two occurs on a set of measure zero and taking the union over $n_{0}, n_{1}$, we get $f \sim g$ as claimed.

NOTATION. In keeping with our idea that the two topoi, $\operatorname{Sh}(X)$ and $\operatorname{Sh}(\mathscr{A} / \mathscr{N})$, are interchangeable, we will also use $M_{Y}(-)$ to denote the similar object of $\operatorname{Sh}(X)$.

Two important special cases are:

EXAmPLe 5. $\mathbf{R}(-):=M_{\mathbf{R}}(-)$ where $(\mathbf{R}, \mathscr{L}, \lambda)$ is the Lebesgue real line. In proposition 4.1, we will show that this is the object of Dedekind reals.

EXAMPLE 6. $\mathbf{C}(-):=M_{\mathrm{C}}(-)$ where $(\mathbf{C}, \mathscr{L} \otimes \mathscr{L}, \lambda \otimes \lambda)$ is the Lebesgue complex plane.

Obvious measure theoretic constructions may not necessarily be interpreted as sheaves, however:

(COUNTER)EXAMPLE 7.

$$
L^{2}(A):=\left\{\left.A_{0} \stackrel{f}{\longrightarrow} \mathbf{C}\left|A_{0} \in \mathscr{A}, \quad A_{0} \subseteq A, \quad \mu\left(A \backslash A_{0}\right)=0, \quad \int_{A}\right| f\right|^{2} d \mu<\infty\right\} / \sim,
$$


does not define sheaf. Let $X:=[0,1], A:=(0,1)$ with cover $A_{n}:=(1 /(n+$ $1), 1 / n$ ) (all with Lebesgue measure) and let $f_{n}(x)=1 / x$. Then, on each piece, $\int_{A}\left|f_{n}\right|^{2} d \mu<\infty$, but extending to $f(x)=1 / x$ on $(0,1)$, we see that $\int_{0}^{1}|f|^{2} d \mu \nless \infty$.

However, $L^{2}(-)$ is a presheaf, $L^{2}(-) \subseteq \mathbf{C}(-)$ as presheaves, and:

PROPOSITION 3.4. $\mathrm{C}(-)$ is the associated sheaf of $L^{2}(-)$.

Proof. Let $A \stackrel{f}{\longrightarrow} \mathbf{C}$ be measurable. We must exhibit a cover of $A$ such that $f \in L^{2}$ on each piece. Let $A_{n}:=\{x|| f(x) \mid<n\}$. Then $A_{n}$ is measurable and $A=\bigcup_{n=1}^{\infty} A_{n}$ and

$$
\int_{A_{n}}|f(x)|^{2} d \mu \leq \int_{A_{n}} n^{2} d \mu=n^{2} \mu\left(A_{n}\right)<n^{2} \mu(X)<\infty
$$

REMARK. In a similar manner, $\mathbf{C}(-)$ is the associated sheaf of all the $L^{p}(-)$ presheaves.

EXAMPLE 8.

$\operatorname{M0R}(A, Y):=\left\{A_{0} \stackrel{f}{\longrightarrow} Y \mid A_{0} \in \mathscr{A}, A_{0} \subseteq A, \mu\left(A \backslash A_{0}\right)=0, f \in \underline{\mathbf{M 0 R}}\right\} / \sim$ defines a sheaf. This is similar to Example 4 above.

If we try disintegrations in a similar manner to Example 8, we do not get a sheaf. Before discussing a counterexample, we give a definition:

DEFINITION 3.2. Let $(X, \mathscr{A}, \mu) \stackrel{\left(f, \mu_{y}\right)}{\longrightarrow}(Y, \mathscr{B}, v)$ and $(X, \mathscr{A}, \mu) \stackrel{\left(g, \eta_{y}\right)}{\longrightarrow}(Y, \mathscr{B}, v)$ be disintegrations. We say $f \sim g$ if two conditions hold. The first is $\mu\{x \mid f(x) \neq$ $g(x)\}=0$. We can restrict $f$ and $g$ to $G:=\{x \mid f(x)=g(x)\}$ to get disintegrations, $\left(G,\left.\mathscr{A}\right|_{G},\left.\mu\right|_{G}\right) \stackrel{\left(\left.f\right|_{G}, \beta_{y}\right)}{\longrightarrow}(Y, \mathscr{B}, v)$ and $\left(G,\left.\mathscr{A}\right|_{G},\left.\mu\right|_{G}\right) \stackrel{\left(\left.g\right|_{G}, \alpha_{y}\right)}{\longrightarrow}(Y, \mathscr{B}, v)$. On $G, f=$ $g$, so $f^{-1}(y) \cap G=g^{-1}(y) \cap G$ for all $y \in Y$. The second condition for $\sim$ is that the measure structures are equal, $\beta_{y}=\alpha_{y}$, for all $y \in Y$.

PROPOSITION 3.5.

$$
\operatorname{Disint}(A, Y):=\left\{\left(f,\left(\left.\mathscr{A}\right|_{A_{0}}\right)_{y},\left(\left.\mu\right|_{A_{0}}\right)_{y}\right): A_{0} \longrightarrow Y \mid f \in \underline{\text { Disint }}\right\} / \sim,
$$

where $\left(A_{0}, f\right) \sim\left(A_{1}, f^{\prime}\right)$ if $\mu\left(A_{0} \triangle A_{1}\right)=0$ and $\left.\left.f\right|_{A_{0} \cap A_{1}} \sim f^{\prime}\right|_{A_{0} \cap A_{1}}$ as disintegrations, defines a presheaf.

PROOF. Restriction of a disintegration to a subspace yields a disintegration (see [7]). 
(COUNTER)EXAmPLe 9. Disint $(-, Y)$ is not a sheaf. This is essentially the same problem as with $L^{2}(-)$. We may choose representatives for a compatible family $\left(C_{n},\left(f_{n},\left(\left.\mu\right|_{C_{n}}\right)_{y}\right)\right)$ where the $C_{n}$ are disjoint. Put $C=\bigcup_{n} C_{n}$ and define $C \stackrel{\left(f,\left(\left.\mu\right|_{C}\right)_{y}\right)}{\longrightarrow} Y$ as $f(x)=f_{n}(x)$ where $n$ is the unique index for which $x \in C_{n}$. Then $f$ is measurable as in Example $4 ;\left(\left.\mu\right|_{C}\right)_{y}$ is a measure for each $y$ and $y \longmapsto\left(\left.\mu\right|_{C}\right)_{y}$ is $v$-measurable. However, if the $\left(\left.\mu\right|_{C_{n}}\right)_{y}$ 's are bounded, there is no guarantee that these are bounded over $n$. Thus, Disint $(-, Y)$ is not a sheaf. But, it almost is; everything works except boundedness. The extension respects $\sim$ and even Axiom 2 holds:

$$
\begin{aligned}
\int_{Y}\left(\mu \mid \cup C_{n}\right)_{y}\left(A \cap \bigcup_{n} C_{n} \cap f_{n}^{-1}(y)\right) d v(y) & =\sum_{n} \int_{Y}\left(\left.\mu\right|_{C_{n}}\right)_{y}\left(A \cap C_{n} \cap f_{n}^{-1}(y)\right) d v(y) \\
& =\sum_{n}\left(\left.\mu\right|_{C_{n}}\left(A \cap C_{n}\right)\right) \\
& =\left.\mu\right|_{\cup C_{n}}\left(A \cap \bigcup_{n} C_{n}\right) .
\end{aligned}
$$

We next give an explicit description of $\operatorname{Sh}(X)$ as a topos over $\underline{\text { Set. }}$.

PROPOSITION 3.6. $\Delta \dashv \Gamma$ in

$$
\operatorname{Sh}(X) \underset{\Gamma}{\stackrel{\Delta}{\leftrightarrows}} \underline{\text { Set }}
$$

where $\Gamma(F \stackrel{\eta}{\longrightarrow} G)=F(X) \stackrel{\eta_{X}}{\longrightarrow} G(X)$ and for $K \in \underline{\text { Set }}$ and $A \in \mathscr{A}$,

$$
\begin{aligned}
\Delta(K)(A)=\{(B, f) \mid & \mu(A \triangle B)=0, B \stackrel{f}{\longrightarrow} K, \\
& \left.f(B) \text { countable, } f^{-1}(k) \in \mathscr{A} \text { for all } k \in K\right\} / \sim,
\end{aligned}
$$

with $(B, f) \sim\left(B^{\prime}, f^{\prime}\right)$ if and only if $\mu\left(B \triangle B^{\prime}\right)=0$ and $\mu\left\{x \in B \cap B^{\prime} \mid f(x) \neq\right.$ $\left.f^{\prime}(x)\right\}=0$.

PROOF. $\Delta(K)$ is the sheafification of a $K$-indexed coproduct (in the presheaf category) of copies of hom $(-, 1)$. To sheafify, take $K$-valued, $(\mathscr{A}, \mu)$-locally constant functions.

REMARK. In particular, this proposition implies $\Delta(K \times L)=\Delta(K) \times \Delta(L)$. We shall implicitly use this when discussing substitution for Hilbert space objects in Section 5.2.

\section{Hilbert sheaves}

4.1. Number systems in $\operatorname{Sh}(\mathbf{X})$ The natural numbers object in $\operatorname{Sh}(X)$ is $\Delta(\mathbf{N})=$ $M_{N}(-)$ since $\Delta$ is a left adjoint $[5$, p. 168]. This is also true for the objects of integers 
and rationals (that is, they are just $\Delta$ applied to the appropriate set). Arithmetic is determined from left exactness of $\Delta$ and is pointwise. For example, for $p, q \in$ $\mathbf{Q}(A)=M_{\mathbf{Q}}(A), q \neq 0, p / q(x)=p(x) / q(x)$. Global constants (over $x$ ) are denoted by enclosure in " $\Gamma\rceil$. '

We noted in Section 3.1 that $S h(X)$ satisfies the axiom of choice. In particular, it satisfies the axiom 'supports split' [5, p.141]. Two interesting applications concerning real numbers arise:

PROPOSITION 4.1. The object of Dedekind reals in $\operatorname{Sh}(X)$ is $\mathbf{R}_{X}(-)$ where

$$
\begin{aligned}
& \mathbf{R}_{X}(A)=M_{\mathbf{R}}(A)=\left\{\left(A_{0}, f\right) \mid \mu\left(A \triangle A_{0}\right)=0\right. \text { and } \\
&\left.\left(A_{0},\left.\mathscr{A}\right|_{A_{0}}\right) \stackrel{f}{\longrightarrow}(\mathbf{R}, \text { Lebesgue }) \text { is measurable }\right\} / \sim .
\end{aligned}
$$

PROOF. ([5, p. 213]) A measurable function, $A_{0} \stackrel{f}{\longrightarrow} \mathbf{R}$ gives a Dedekind cut, $(L, U)$, by defining $L:=\left\{q \in \mathbf{Q} \mid q(x)<f(x)\right.$ on $\left.A_{0}\right\}$ and $U:=\{q \in \mathbf{Q} \mid f(x)<q(x)\}$.

Conversely, given a Dedekind cut $(L, U)$, 'supports split' allows us to choose sequences $q_{n} \in L$ and $q^{n} \in U$ with $q^{n}-q_{n}<1 / n$. For almost all $x$, these two sequences tend to a common limit $f(x) \in \mathbf{R}_{X}(A)$.

NOTATION. $\mathbf{R}_{c}$ denotes the object of Cauchy reals (that is, equivalence classes of Cauchy sequences in $\mathbf{Q}_{X}$; see [5, p. 218]).

PROPOSITION 4.2. $\mathbf{R}_{c} \cong \mathbf{R}_{X}$ (that is, the Cauchy and Dedekind reals coincide) in $\operatorname{Sh}(X)$.

Proof. We claim the canonical inclusion, with components $\mathbf{R}_{c}(A) \stackrel{j_{A}}{\rightarrow} \mathbf{R}_{X}(A)$, $A \in \mathscr{A}$, is an isomorphism. Let $r=(L, U)$ be a Dedekind real. $\operatorname{Sh}(A)$ satisfies 'supports split' so choose a sequence of sections $\left(q_{n}, q^{n}\right) \in \mathbf{Q}_{A} \times \mathbf{Q}_{A}$ such that $q_{n} \in L$, $q^{n} \in U$, and $q^{n}-q_{n}<1 / n$. Then $\left\langle q_{n}\right\rangle \in \mathbf{R}_{c}(A)$ and $j_{A}\left\langle q_{n}\right\rangle=r$ so $j_{A}$ is onto as required. And so, $j$ is an isomorphism.

REMARK. (1) The proof above is similar to [5, Example 6.68] where it is shown that $\mathbf{R}_{c} \cong \mathbf{R}_{X}$ in the category of sheaves on a separable zero-dimensional topological space.

(2) Various entities of $\mathbf{Q}, \mathbf{R}$, etcetera are easily described in terms of functions. For example, the order used for $L$ in Proposition 4.1 is ' $f(x)<g(x)$ on $A$ ' which means ' $f(x)<g(x)$ for almost all $a \in A^{\prime},<$ is an internal order. That is, $\mathbf{R} \times \mathbf{R} \cong \mathbf{R}+<+$ $>\left(\right.$ for $(f, g) \in(\mathbf{R} \times \mathbf{R})(A), A_{1}=\{x \mid f(x)=g(x)\}, A_{2}=\{x \mid f(x)<g(x)\}$, and $A_{3}=\{x \mid f(x)>g(x)\}$ forms a cover). 
$\mathbf{C}(-)=M_{\mathbf{C}}(-)$ is the complex numbers object. Taking the real or imaginary part of a complex-valued, measurable function yields a real-valued, measurable function and we have $\mathbf{C}(-) \subseteq \mathbf{R}(-) \times \mathbf{R}(-)$. In [6], Rousseau notes that a $\mathbf{C} \cong \mathbf{R} \times \mathbf{R}$ is a suitable complex numbers object in any topos for which $\mathbf{R}_{c}$ is complete (see also Section 4.3 for a discussion on completeness).

It is a straightforward matter to define operations which give $\mathbf{C}(-)$ the structure of a ring with involution. It satisfies the axiom of non-triviality [3] and is, in fact a geometric field (in which case, see [3], it will also be a field of fractions and a field of quotients since $\operatorname{Sh}(X)$ is Boolean):

PROPOSITION 4.3. $\mathrm{C}(-)$ is a geometric field.

PROOF. The group of units is

$$
\begin{aligned}
U(A) & =\left\{\left(A_{0}, f\right) \in \mathbf{C}(A) \mid \exists\left(A_{1}, g\right) \in \mathbf{C}(A),\left(A_{0}, f\right) \cdot\left(A_{1}, g\right) \sim\lceil 1\rceil\right\} \\
& =\left\{\left(A_{0}, f\right) \in \mathbf{C}(A) \mid \mu\left\{x \in A_{0} \mid f(x)=0\right\}=0\right\} .
\end{aligned}
$$

We must show that $\mathbf{1} \stackrel{\lceil 0\rceil}{\longrightarrow} \mathbf{C} \longleftarrow U$ is a coproduct diagram. Specifically, we must show for an $f \in \mathbf{C}(A)$, there is a cover $\left\{A_{i} \hookrightarrow A\right\}$ such that $\left.f\right|_{A_{i}} \in U\left(A_{i}\right)$ or $\left.f\right|_{A_{i}} \sim 0$. Consider the two sets $A_{z}=\left\{a \in A_{0} \mid f(a)=0\right\}$ and $A_{n}=\left\{a \in A_{0} \mid f(a) \neq 0\right\}$. $\left\{A_{2}, A_{n}\right\}$ forms a cover of $A$. Furthermore, $\left.f\right|_{A_{z}}=0$ and $f \mid A_{n} \in U\left(A_{n}\right)(1 / f$ is measurable on $A_{n}$ and will be the inverse $g$ ).

\subsection{A sheaf from a measurable field}

Definition 4.1 ([1]). Let $(X, \mathscr{A}, \mu)$ be a measure space. A measurable field of Hilbert spaces or MFHS, $\left(H(x)_{x \in X}, \mathscr{G}\right)$, is a family of separable Hilbert spaces and a subset $\mathscr{G} \subseteq \prod_{x \in X} H(x)$ which is subject to the axioms:

(1) $x \mapsto\|g(x)\|$ is measurable for each $g \in \mathscr{G}$,

(2) if $h(x) \in \prod_{x \in X} H(x)$ has the pointwise inner product, $x \mapsto\langle h(x), g(x)\rangle$, measurable for all $g \in \mathscr{G}$, then $h \in \mathscr{G}$, and

(3) there is a sequence $\left\langle g_{i}\right\rangle_{i=1}^{\infty}$ of elements of $\mathscr{G}$ such that for each $x, \operatorname{Span}\left\{g_{i}(x) \mid\right.$ $i=1,2,3, \ldots\}$ is dense in $H(x)$.

The elements of $\mathscr{G}$ are called measurable fields of vectors or MFV's and the sequence in Axiom 3 is called a fundamental sequence.

In this section, we describe a sheaf, $G$, to be constructed from a measurable field of Hilbert spaces. We will use $G$ and $\mathbf{C}$ as motivating examples for Hilbert sheaves.

PROPOSITION 4.4. $G(A):=\{g \in \mathscr{G} \mid g(x)=0 \forall x \notin A\} / \sim$. defines a presheaf $G(-):(\mathscr{A}, \subseteq)^{o p} \longrightarrow$ Set. 
ProOF. For $A^{\prime} \subseteq A$, restriction is given by $G(A) \longrightarrow G\left(A^{\prime}\right),(g(x))_{x \in X} \mapsto$ $\left(g^{\prime}(x)\right)_{x \in X}$, where

$$
g^{\prime}(x)= \begin{cases}g(x) & x \in A^{\prime} \\ 0 & \text { else. }\end{cases}
$$

Note that $\left(x \mapsto\left\langle h(x) \mid g^{\prime}(x)\right\rangle\right)=\left(x \mapsto\langle h(x) \mid g(x)\rangle \cdot \chi_{A^{\prime}}\right)$ is measurable for all $h \in \mathscr{G}$, whence, by axiom $2, g^{\prime} \in \mathscr{G}$.

PROPOSITION 4.5. $G(-)$ is a sheaf.

PROOF. The proof is similar to that for Proposition 3.3. The only question is whether $g$, the unique extension of a compatible family $\left\{g_{i}\right\}$ on a cover $\left\{A_{i}\right\}$ of $A$ is in $\mathscr{G}$. But $x \mapsto\left\langle h(x) \mid g_{i}(x)\right\rangle$ is measurable for each $g_{i}$ and for all $h \in \mathscr{G}$ since each $g_{i}$ is in $\mathscr{G}$. Apply Axiom 2 for $\mathscr{G}$.

We can make $G(A)$ into a $\mathbf{C}(A)$-module by defining operations pointwise. $G$ is a $\mathrm{C}$-vector space and can be made into a normed vector space. The sheaf of non-negative reals is given at $A$ by

$$
\mathbf{R}^{+}(A)=\left\{A \stackrel{f}{\longrightarrow} \mathbf{R}^{+} \text {measurable }\right\} / \sim .
$$

For each $A$, the function $G(A) \stackrel{\|\cdot\|_{A}}{\longrightarrow} \mathbf{R}^{+}(A)$ given by $g \longmapsto\|g\|$, where $\|g\|(x)=$ $\|g(x)\|_{H(x)}$, is measurable by Axiom 1 for an MFHS. Then $g \mapsto\|g\|$ is well-defined since if $g=g^{\prime}$ except on $B$ with $\mu(B)=0$, then $\|g\|=\left\|g^{\prime}\right\|$ except on $B$ as well. Since $\|\cdot\|$ is natural in $A$, we have a map $G \stackrel{\|\cdot\|}{\longrightarrow} \mathbf{R}^{+}$in $\operatorname{Sh}(X)$. The norm axioms follow from those for the $H(x)$ 's.

4.3. Hilbert sheaves (definitions and topology) We use the above discussion about $G, \mathbf{C}$, and the norm, as motivation for our notion of Hilbert space object in $S h(X)$. In this section, we define such and discuss topological notions such as completeness.

Recall, there are two equivalent ways to describe distance in a Hilbert space. One is to give a positive definite inner product, $\langle-\mid-\rangle$, which yields a norm (via $\|\cdot\|=\sqrt{(\cdot \mid \cdot)}$ ) that satisfies the parallelogram law,

$$
\|f+g\|^{2}+\|f-g\|^{2}=2\|f\|^{2}+2\|g\|^{2} .
$$

Another way is to give a norm that satisfies the parallelogram law and define an inner product using the polarization identity,

$$
\langle f \mid g\rangle=\frac{1}{4}\|f+g\|^{2}-\frac{1}{4}\|f-g\|^{2}+\frac{i}{4}\|f+i g\|^{2}-\frac{i}{4}\|f-i g\|^{2} .
$$


Now, suppose $H$ is an inner product space over $\mathbf{C}$ in $S h(X)$. We have natural transformations: $H \times H \stackrel{(-1-)}{\longrightarrow} \mathbf{C}$ and $H \stackrel{\|\cdot\|}{\longrightarrow} \mathbf{R}^{+}$. These are to satisfy the obvious axioms (the classical ones translated as equations for morphisms). For example, positive definiteness may be regarded as the existence of a factorization of

$$
H \stackrel{\Delta}{\longrightarrow} H \times H \stackrel{(\cdot)}{\longrightarrow} \mathrm{C}
$$

through $\mathbf{R}^{+}$considered as a sub-sheaf of $\mathbf{C}$. An inner product yields a norm and a norm yields an inner product as in the classical case.

DEFINITION 4.2. A pre-Hilbert space object in $S h(X)$ is a positive definite inner product space over $C$. A morphism between two such is a natural transformation $H(A) \stackrel{\tau_{A}}{\longrightarrow} K(A)$, which is linear $\left(\tau_{A}\left(f+_{H(A)} g\right)=\tau_{A}(f)+_{K(A)} \tau_{A}(g)\right)$ and bounded (there is a $b \in \mathbf{R}_{X}^{+}$such that $\forall h \in H,\|\tau(h)\|_{K} \leq b\|h\|_{H}$ ). This gives a category which we denote by $\operatorname{Pre}(S h(X))$.

REMARK. If $\tau$ is bounded, we can find a $b \geq 1$ (in particular, bounded away from zero) such that $\|\tau(h)\| \leq b\|h\|$. Furthermore, the restrictions $\rho_{A^{\prime}}^{A}$ are linear and bounded (by 1 ).

We next discuss completeness.

DEFINITION 4.3. For $F$ in $S h(X)$, a sequence in $F$ is a map $\mathbf{N}_{X} \stackrel{S}{\longrightarrow} F$.

REMARK. In a Grothendieck topos, $\mathbf{N}_{X}=\sum_{n \in \mathbf{N}} 1_{X}$, so a sequence is simply a sequence of global elements (that is, a function $\mathbf{N} \longrightarrow F(X)$ ).

DEFINITION 4.4. Let $(F, d)$ be a metric space in $S h(X)$.

(i) The sequence $\mathbf{N} \stackrel{\left\langle s_{n}\right\rangle}{\longrightarrow} F(X)$ is said to be convergent if $\exists s \in F(X)\left(\forall k \in \mathbf{N}_{X}^{+}(\exists\right.$ a cover $\left\{A_{i}\right\}_{i=1}^{\infty}$ of $X$ and $\exists N_{i}, i=1,2,3, \ldots$, such that $\forall n \geq N_{i}, d\left(s_{n}, s\right)<$ $1 / k$ on $\left.A_{i}\right)$ ).

(ii) The sequence $\mathbf{N} \stackrel{\left(s_{n}\right)}{\longrightarrow} F(X)$ is said to be Cauchy if $\forall k \in \mathbf{N}_{X}^{+}\left(\exists\right.$ a cover $\left\{A_{i}\right\}_{i=1}^{\infty}$ of $X$ and $\exists N_{i}, i=1,2,3, \ldots$, such that $\forall n, m \geq N_{i} d\left(s_{n}, s_{m}\right)<1 / k$ on $\left.A_{i}\right)$.

REMARK. (1) $A$-convergent and $A$-Cauchy can be defined as the above with $X$ replaced by $A$.

(2) If $X=1$, these are the usual notions for ordinary metric spaces.

Definition 4.5. $(F, d)$ is said to be complete if every Cauchy sequence in $F$ converges in $F$. 
PROPOSITION 4.6. $\mathbf{R}_{X}$ with its norm-induced metric is complete.

REMARK. The classical proof that $\mathbf{R}$ is Cauchy complete involves a sequence of steps: (1) Cauchy implies bounded, (2) sequence implies $\exists$ monotone subsequence, (3) monotone sequence and bounded implies convergent, and (4) Cauchy and convergent subsequence implies convergent. This does not translate to our case. For example, if $s_{n} \rightarrow s$ pointwise, then we do not necessarily have a subsequence that increases to $s$. Proposition 4.6 will be proved in two steps: (1) $s_{n}$ Cauchy implies $\exists s, s_{n} \rightarrow s$ pointwise and (2) $s_{n} \rightarrow s$ pointwise implies $s_{n} \rightarrow s$. We state these as lemmas for future reference.

LEMMA 4.1. Let $\left\langle s_{n}\right\rangle$ be a Cauchy sequence in $\mathbf{R}_{X}$. Then there is an $s \in \mathbf{R}_{X}$ such that $s_{n} \rightarrow s$ pointwise.

PROOF. Let $\left\langle s_{n}\right\rangle$ be a Cauchy sequence. Then, by definition, for each $k \in \mathbf{N}_{X}^{+}$, there is a cover $\left\{A_{i}\right\}$ and $N_{i}$, such that $\forall n, m \geq N_{i},\left(\left\|s_{n}(x)-s_{m}(x)\right\|<1 / k\right.$ on $\left.A_{i}\right)$. In particular, $\left\langle s_{n}(x)\right\rangle$ is a Cauchy sequence for almost all $x \in X$ (we can choose $k$ to be constant). $\mathbf{R}$ is complete, so there is an $s(x)$ such that $s_{n}(x) \rightarrow s(x)$. Since $s$ is the pointwise limit of measurable functions, it is measurable and there is an $N$ such that $\left\|s(x)-s_{N}(x)\right\|<\lceil 1\rceil$. Now, $\left\|s_{N}(x)\right\|<\infty$, since $s_{N} \in \mathbf{R}_{X}$, which implies $\|s(x)\|<1+\left\|s_{N}(x)\right\|<\infty$ so $s \in \mathbf{R}_{X}$.

LEMMA 4.2. $s_{n}(x) \rightarrow s(x)$ pointwise implies $s_{n} \rightarrow \sin \mathbf{R}_{X}$.

Proof. Suppose $s_{n}(x) \rightarrow s(x)$ pointwise. Let $k \in \mathbf{N}_{X}^{+}$. We seek a cover $\left\{A_{i}\right\}_{i=1}^{\infty}$ of $X$ and $N_{i}$ such that $\forall n>N_{i}\left(\left\|s_{n}-s\right\|<1 / k\right.$ on $\left.A_{i}\right)$ (Definition 4.4).

Assume first that $k=\lceil k\rceil$ is constant. Let $G_{n}=\left\{x \mid\left\|s_{n}(x)-s(x)\right\|<1 /\lceil k\rceil\right\}$ and $E_{i}=\bigcap_{n=i}^{\infty} G_{n}=\left\{x \mid\left\|s_{n}(x)-s(x)\right\|<1 /\lceil k\rceil\right.$ for all $\left.n \geq i\right\}$. Suppose $x \in A$; then since $s_{n}(x) \rightarrow s(x)$, there is an $N$ such that $\left\|s_{n}(x)-s(x)\right\|<1 /\lceil k\rceil$ for $n \geq N$. That is, $x \in E_{N}$ for some $N$. Thus, the $E_{i}$ 's cover $A$. Put $N_{i}=i$ and we have found our cover and the $N_{i}$ for which $\left\|s_{n}-s\right\|<1 /\lceil k\rceil$. Now suppose $k \in \mathbf{N}_{X}^{+}$is locally constant. By considering $A_{j}=\{x \mid k(x)=j\}$ and applying the above special case to each $A_{j}, s_{n} \rightarrow s$ as required.

DEFINITION 4.6. A Hilbert space object or Hilbert sheaf in $S h(X)$ is an inner product space over $\mathbf{C}_{X}$ which is complete in the induced norm.

PROPOSITION 4.7. The pre-Hilbert sheaf constructed from an MFHS is complete.

PROOF. The proof is exactly as that for the completeness of $\mathbf{R}$. The only issue is whether the pointwise limit, $s(x)=\lim _{n \rightarrow \infty} s_{n}(x)$, is in $\mathscr{G}$. But, for all $g \in \mathscr{G}, x \mapsto$ 
$\langle s(x) \mid g(x)\rangle=\lim _{n \rightarrow \infty}\left(x \mapsto\left\langle s_{n}(x) \mid g(x)\right\rangle\right)$ is measurable. By Axiom 2 for measurable fields, $s \in \mathscr{G}$ as required.

COROllary. $\mathbf{C}(-)$ is complete.

We end this section with a discussion about the completion of a pre-Hilbert space object. Many of the proofs mimic classical ones so will be omitted. They require some translation into the language of sheaves but this is not difficult. As an example of the techniques used, we prove Lemma 4.3. It exhibits an ' $\epsilon / 2$ proof' in this context. Ultimately, we will describe a functor

$$
\underline{\operatorname{Pre}}(\operatorname{Sh}(X)) \stackrel{c()}{\longrightarrow} \underline{\operatorname{Hilb}}(\operatorname{Sh}(X)) .
$$

Definition 4.7. Let $H \in \operatorname{Pre}(\operatorname{Sh}(X))$. The completion of $H, c(H)(A)$, is the set of equivalence classes of $A$-Cauchy sequences with $\left\langle s_{n}\right\rangle \equiv\left\langle t_{n}\right\rangle$ if and only if $\lim _{n \rightarrow \infty}\left\|s_{n}-t_{n}\right\|=0$ (this latter limit taken in $\mathbf{R}^{+}(A)$ ).

\section{LEMMA 4.3. The relation ' $\equiv$ ' is an equivalence relation.}

PROOF. Certainly, $\equiv$ is reflexive and symmetric: $\left(-\left(s_{n}-t_{n}\right)=\left(t_{n}-s_{n}\right)\right.$ and $\|(-1) h\|=\|-1\|\|h\|=\|h\|)$. Now suppose $\left\|s_{n}-t_{n}\right\| \rightarrow 0$ and $\left\|t_{n}-u_{n}\right\| \rightarrow 0$ in $\mathbf{R}^{+}(A)$. Let $k \in \mathbf{N}_{X}^{+}$. There is a cover $\left\{A_{i}\right\}$ of $A$ and $\exists M_{i}\left(\forall n \geq M_{i}\left\|s_{n}-t_{n}\right\|<1 /(\lceil 2\rceil k)\right.$ on $\left.A_{i}\right)$ and there is a cover $\left\{A_{j}^{\prime}\right\}$ of $A$ and $\exists N_{j}\left(\forall n \geq N_{j}\left(\left\|t_{n}-u_{u}\right\|<1 /\lceil 2\rceil k\right.\right.$ on $\left.\left.A_{j}^{\prime}\right)\right)$. Let $P_{i j}=\max \left\{M_{i}, N_{j}\right\}$ and $B_{i j}=A_{i} \cap A_{j}^{\prime}$. Then $\left\{B_{i j}\right\}$ is a cover of $A$ and

$$
\left\|s_{n}-u_{n}\right\|=\left\|s_{n}-t_{n}+t_{n}-u_{n}\right\| \leq\left\|s_{n}-t_{n}\right\|+\left\|t_{n}-u_{n}\right\|<\frac{1}{\lceil 2\rceil k}+\frac{1}{\lceil 2\rceil k}=\frac{1}{k}
$$

for all $n \geq P_{i j}$ on $B_{i j}$.

$c(H)(-)$ is a sheaf and operations on $c(H)(A)$ are defined pointwise: $0=\langle 0\rangle_{n=1}^{\infty}$, $-\left\langle s_{n}\right\rangle=\left\langle-s_{n}\right\rangle,\left\langle s_{n}\right\rangle+\left\langle t_{n}\right\rangle=\left\langle s_{n}+t_{n}\right\rangle, \alpha \cdot\left\langle s_{n}\right\rangle=\left\langle\alpha \cdot s_{n}\right\rangle$. These operations are welldefined with respect to $\equiv$. For example, suppose $\left\langle s_{n}\right\rangle \equiv\left\langle s_{n}^{\prime}\right\rangle$ and $\left\langle t_{n}\right\rangle \equiv\left\langle t_{n}^{\prime}\right\rangle$; then $\left\|\left(s_{n}+t_{n}\right)-\left(s_{n}^{\prime}+t_{n}^{\prime}\right)\right\| \leq\left\|s_{n}-s_{n}^{\prime}\right\|+\left\|t_{n}-t_{n}^{\prime}\right\| \longrightarrow 0$ (as in the ' $\epsilon / 2$-proof' of Lemma 4.3). Furthermore, we may define a norm on $c(H)(A)$ by $\left\|\left(s_{n}\right\rangle\right\|=\lim _{n \rightarrow \infty}\left\|s_{n}\right\|$. The following summarize properties of limits and $\|\cdot\|$ :

LEMMA 4.4. $\operatorname{In} \mathbf{R}_{X}$,

(1) $a \rightarrow a$

(2) $a_{n} \rightarrow a, a_{n}$ positive implies a nonnegative

(3) $a_{n}-b_{n} \rightarrow 0, a_{n} \rightarrow a$ implies $b_{n} \rightarrow a$ 
(4) $a_{n} \rightarrow a, b_{n} \rightarrow$ b implies $a_{n}-b_{n} \rightarrow a-b$

(5) $a_{n} \rightarrow a, b_{n} \rightarrow b, a_{n}<b_{n}$ implies $a \leq b$

(6) $\mathbf{R} \stackrel{\tau}{\longrightarrow} \mathbf{R}$ bounded, $a_{n} \rightarrow$ a implies $\tau\left(a_{n}\right) \rightarrow \tau(a)$.

LEMMA 4.5. $\|\cdot\|$ is well defined and a norm on $c(H)(A)$.

LEMMA 4.6. (a) Let $H^{c}(A)=\left\{\langle s\rangle_{n=1}^{\infty} \mid s \in H(A)\right\} / \equiv$ (that is, equivalence classes of constant sequences). Then $H^{c}(A)$ is isometric to $H(A)$ and closure $\left(H^{c}(A)\right)=c(H)(A)$.

(b) Cauchy sequences $H^{c}(A)$ converge in $c(H)(A)$.

(c) Cauchy sequences in $c(H)(A)$ converge in $c(H)(A)$.

And so, we only need to prove the uniqueness part of the following theorem.

THEOREM 4.1. For $H$ a pre-Hilbert sheaf, there is a Hilbert sheaf, $c(H)$, which contains a dense, isometric copy of $H$. Furthermore, if $K$ is another Hilbert sheaf with this property, then $K$ is isometric to $c(H)$.

Proof. Suppose $H \cong H^{c} \subseteq c(H)$ and $H \cong H^{2} \subseteq K$ with $H^{c} \stackrel{\phi}{\longrightarrow} H^{2}$ an isometric isomorphism. The isometry between $c(H)$ and $K$ is defined as follows: Given $\left\langle h_{n}\right\rangle \in c(H)$, put $k=\lim _{n \rightarrow \infty} \phi\left(h_{n}\right)$. Conversely, given $k \in K$, let $h_{n} \rightarrow k$ with $h_{n} \in H^{2}$. We get $\left\langle\phi^{-1}\left(h_{n}\right)\right\rangle \in c(H)$.

DEFINITION 4.8. $c(H)$ is called the completion of $H$.

For $H \stackrel{T}{\longrightarrow} K$ in $\underline{\operatorname{Pre}}(S h(X))$, define $c(H) \stackrel{c(T)}{\longrightarrow} c(K)$ by $c(T)\left\langle s_{n}\right\rangle=\left\langle T\left(s_{n}\right)\right\rangle$. Then $\left\langle s_{n}\right\rangle$ Cauchy implies $\left\langle T\left(s_{n}\right)\right\rangle$ Cauchy and $\left\langle s_{n}\right\rangle \equiv\left\langle t_{n}\right\rangle$ implies $\left\langle T\left(s_{n}\right)\right\rangle \equiv\left\langle T\left(t_{n}\right)\right\rangle$. And so, there is a functor (which, in fact, is left adjoint to the forgetful functor):

$$
\underline{\text { Pre }}(S h(X)) \stackrel{c()}{\longrightarrow} \underline{\text { Hilb }}(S h(X)) \text {. }
$$

REMARK. In Proposition 4.2, we showed that the Cauchy and Dedekind reals coincide in $S h(X)$. In view of the above completion process, we note that the Cauchy formulation is more useful for our purposes.

Another useful result which we state without proof (it is simply another $\epsilon / 2$ argument) is the following:

PROPOSITION 4.8. $c(-)$ is product preserving.

REMARK. This proposition says that algebraic constructions on a normed space get transported to its completion. For example, this is the essence of much of what is said in Lemma 4.4. 


\section{Application to measure indexing}

5.1. The direct integral We begin by describing the direct integral of a Hilbert sheaf and will generalize below. Let $H \in \underline{\mathbf{H i l b}}(S h(X))$, and define

$$
\int^{\oplus} H=\left\{s: 1 \rightarrow H(X) \mid \int\|s\|_{X}^{2} d \mu<\infty\right\} .
$$

REMARK. Since we are working in $\operatorname{Sh}(X)$, we must specify that the above integral is finite for any choice of representative of $\|s\|$ (it is an element of $\mathbf{R}$ so may be considered as an equivalence class). It is easy to show that 'for any choice' may be replaced by 'for some choice'. We note that such choices are part of the price to be paid when working with the more index-oriented setting, $\operatorname{Sh}(X)$, as opposed to $\operatorname{Sh}(\mathscr{A} / \mathscr{N})$.

Operations on $\int^{\oplus} H$ are inherited from those on $H$. We may define an inner product in an obvious way:

DEFINITION 5.1. For $s, t \in \int^{\oplus} H$, define their inner product as $\langle s \mid t\rangle_{2}:=\int\langle s \mid t\rangle_{X} d \mu$. The resulting norm is called the $\|\cdot\|_{2}$ norm.

THEOREM 5.1. $\int^{\oplus} H$ is complete in the $\|\cdot\|_{2}$ norm.

PROOF. Let $\left\langle s_{n}\right\rangle$ be a 2-Cauchy sequence in $\int^{\oplus} H$. We can choose a subsequence, also called $\left\langle s_{n}\right\rangle$, such that $\sum_{n=1}^{\infty}\left\|s_{n+1}-s_{n}\right\|_{2}<\infty$. We claim that $t_{N}=s_{1}+$ $\sum_{n=1}^{N}\left(s_{n+1}-s_{n}\right)$ converges to a $t \in H$ and $t=\lim _{n \rightarrow \infty} s_{n} \in \int^{\oplus} H$. To prove this, we must show that $t_{N}$ is a Cauchy sequence. That is, for each $k \in \mathbf{N}_{X}^{+}$, we seek a cover, $\left\{A_{i}\right\}_{i=1}^{\infty}$ of $A$, and an $N_{i}$ such that $\forall n, m \geq N_{i}\left(\left\|t_{n}-t_{m}\right\|<1 / k\right.$ on $\left.A_{i}\right)$ (Definition 4.4).

Suppose first that $k$ is a constant. Then $\sum_{n=1}^{\infty}\left\|s_{n+1}-s_{n}\right\|_{2}<\infty$ implies $\sum_{n=1}^{\infty} \| s_{n+1}-$ $s_{n} \|(x)<\infty$ for almost all $x \in X$. Put $A_{M, k}=\left\{x \mid \sum_{n=M}^{\infty}\left\|s_{n+1}-s_{n}\right\|(x)<1 / k\right\}$. Then $\left\{A_{M, k}\right\}_{M=1}^{\infty}$ forms a cover of $X$ and $\left\|t_{p}-t_{q}\right\|<1 / k$ for all $p, q \geq M$. So $t_{N}$ is Cauchy. For a general, locally constant $k$, put $A_{j}=\{x \mid k(x)=j\}$. Then $A_{M, j} \cap A_{j}$ is the required cover and $M$ is the required ' $N_{i}$ ' of Definition 4.4. So $t_{N}$ is Cauchy.

Since $H$ is complete, $t_{N} \rightarrow t=s_{1}+\sum_{n=1}^{\infty}\left(s_{n+1}-s_{n}\right)$ and $\left\|t_{N}-t\right\|_{2} \leq \sum_{n=N}^{\infty} \| s_{n+1}-$ $s_{n} \|_{2} \rightarrow 0$ as $N \rightarrow \infty$ so $t_{N} \rightarrow_{2} t$ as well. Furthermore, $\|t\|_{2} \leq\left\|s_{1}\right\|_{2}+\sum_{n=1}^{\infty} \| s_{n+1}-$ $s_{n} \|_{2}<\infty$ so $t \in \int^{\oplus} H$.

REMARK. $t_{N}$ and $t$ are special in the above. In general, $u_{n} \rightarrow u$ does not imply $u_{n} \rightarrow_{2} u$ (if $\left\|u_{N_{i}}-u\right\|(x)<1 / k$ on $A_{i}$, then we do not necessarily have $\left\|u_{N}-u\right\|_{2}<\epsilon$, say, on all of $X$; the $N_{i}$ 's may increase (over $i$ ) without bound). In order to ensure 2-convergence, we would require some uniformity (a common bound) of the $N_{i}$ 's. However, if $u_{n} \rightarrow u$ and $u_{n}, u \in \int^{\oplus} H$, then $u_{n} \rightarrow_{2} u$ if and only if $\left\|u_{n}\right\|_{2} \rightarrow\|u\|_{2}$. 
A straightforward result is:

PROPOSITION 5.1.

$$
\underline{\mathbf{U H i l b}}(\operatorname{Sh}(X)) \stackrel{f^{\oplus}}{\longrightarrow} \underline{\text { Hilb }}
$$

is a functor where the objects of $\underline{\mathbf{U H i l b}}(S h(X))$ are Hilbert sheaves and the morphisms are uniformly bounded natural linear transformations (that is, $H \stackrel{\tau}{\longrightarrow} K$ linear and for which there is a constant $b$ such that $\left.\forall h \in H,\|\tau(h)\|_{K} \leq b\|h\|_{H}\right)$.

REMARKS. (1) For $H \stackrel{\tau}{\longrightarrow} K$ a bounded (by $b \in \mathbf{R}_{X}^{+}$, say, which is not necessarily a constant) linear transformation and $s \in \int^{\oplus} H, \int\|\tau(s)\|_{X}^{2} d \mu \leq \int\|b\|_{X}^{2}\|s\|_{X}^{2} d \mu$. There is, however, no guarantee that this second integral is finite. So, bounded linear transformations are not adequate to make $\int^{\oplus}$ functorial. We need a stronger condition on the bound.

(2) The set of square-integrable sections of $H(A)$ has a Hilbert space structure, $\int_{A}^{\oplus} H$, for each $A \in \mathscr{A}$. And so, we get an element of $\prod_{A \in \mathscr{A}} H(A)$. This $\mathscr{A}$-family is not arbitrary though, in view of the fact that the restrictions $\rho_{A^{\prime}}^{A}$ are uniformly bounded linear transformations.

5.2. Substitution We next look at substitution and will consider the special case of $\Delta$ first. Recall from Section 3.2, for $K \in \underline{\text { Set}}$,

$$
\begin{aligned}
\Delta(K)(A)=\{(B, f) \mid & \mu(A \triangle B)=0, B \stackrel{f}{\longrightarrow} K, \\
& \left.f(B) \text { countable, } f^{-1}(k) \in \mathscr{A} \forall k \in K\right\} / \sim
\end{aligned}
$$

gives a sheaf in $\operatorname{Sh}(X)$. For $H \in \underline{\text { Hilb}}$, operations on $\Delta(H)(A)$ are the obvious ones: $0 \in \Delta(H)(A)=A \stackrel{\lceil 0\rceil}{\longrightarrow} H ;-(B, f)=(B,-f) ;(B, f)+\left(B^{\prime}, f^{\prime}\right)=(B \cap$ $\left.B^{\prime}, f+f^{\prime}\right)$. These make $\Delta(H)(A)$ into an additive group. However, for $\alpha \in \mathbf{C}_{X}$ and $(B, f) \in \Delta(H)(A), \alpha(x) \cdot f(x)$ does not necessarily have a countable image. Thus, $\Delta(H)$ is not even a $\mathbf{C}_{X}$-vector space (let alone a Hilbert space). Incidentally, that $\Delta$ (Hilbert) $\neq$ Hilbert is not entirely surprising since $\Delta$ is not logical $(\operatorname{Sh}(X)$ is not atomic if, for example, $X$ has no atoms). It does preserve finite products but not necessarily logically more complicated entities like $\mathbf{C}$.

Let $\mathbf{C}_{l c}=\Delta(\mathbf{C})$ be the set of equivalence classes of locally constant $\mathbf{C}$-valued functions. $\mathbf{C}_{l c}$ is a geometric field in a similar manner to $\mathbf{C}_{X}$ (see Proposition 4.3). Then $\Delta(H)$ is a $\mathbf{C}_{l c}$-vector space. Put $\|(B, f)\|:=(B,\|f\|) \in \Delta(\mathbf{R}) \subseteq \mathbf{R}_{X}$. This satisfies positive definiteness and the triangle inequality. As one might expect, this is not a complete norm but the last containment is 'dense' in the following sense: 
Proposition 5.2. Every $f \in \mathbf{C}_{X}$ is the pointwise limit of some sequence in $\Delta(\mathbf{C})(X)$.

PROOF. This is simply the statement that a measurable function is the limit of functions with countable image. $f \in \mathbf{C}_{X}$ can be written as $f=g+i h$, where $g, h \in \mathbf{R}_{X}$ and $g$ and $h$ are each the difference of two non-negative functions. Thus, let $f(x) \geq 0$ and consider

$$
\begin{aligned}
A_{n} & =\{x \mid n-1 \leq f(x)<n\} \quad(n=1,2,3, \ldots) \quad \text { and } \\
A_{n k} & =\left\{x \in A_{n} \mid \frac{1}{k+1} \leq f(x)-(n-1)<\frac{1}{k}\right\} \quad(k=1,2,3, \ldots)
\end{aligned}
$$

and put $f_{N K}(x)=\sum_{n=1}^{N} \sum_{k=1}^{K}((n-1)+1 /(k+1)) \chi_{A_{n k}}$. Then $f_{N K} \rightarrow f$ pointwise.

There is a substitution functor $\operatorname{Sh}(X) \stackrel{\phi^{*}}{\stackrel{S h}{ }} \operatorname{Sh}(Y)$ for each $(X, \mathscr{A}, \mu) \stackrel{\phi}{\longrightarrow}(Y, \mathscr{B}, \nu)$

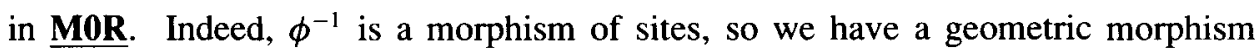
$\phi^{*} \dashv \phi_{*}$ where, for $G \in \operatorname{Sh}(X), A \in \mathscr{A}$, and $a$ the associated sheaf functor,

$$
\phi^{*}(G)(A)=a\left(\operatorname{colim}_{A \subseteq \phi^{-1}(B)} G(B)\right)
$$

and for $F \in S h(X)$ and $B \in \mathscr{B}$,

$$
\phi_{*}(F)(B)=F\left(\phi^{-1}(B)\right) .
$$

As we have noted, $\phi^{*}$ does not preserve Hilbert space objects. However, the proof of Lemma 4.2 and Proposition 5.2 actually give:

PROPOSITION 5.3. $c\left(\Delta_{X} \mathbf{C}\right)=\mathbf{C}_{X}$ (the completion of the sheaf of locally constant functions is the sheaf of all measurable functions).

And so, substitution, $\underline{\text { Hilb }}(S h(X)) \stackrel{\phi^{*}}{\longleftarrow} \underline{\text { Hilb }}(S h(Y))$, may be defined as the triple composite:

$$
\underline{\text { Hilb }}(S h(X)) \stackrel{c}{\longleftarrow} \underline{\operatorname{Pre}}(S h(X)) \stackrel{\phi^{*}}{\longleftarrow} \underline{\operatorname{Pre}}(S h(Y)) \stackrel{u}{\longleftarrow} \underline{\operatorname{Hilb}}(S h(Y))
$$

We next give a brief outline to show that this makes sense (that is, that $\phi^{\#}$ preserves Hilbert space objects). Many of the proofs are left to the reader. $\phi^{*}$ preserves finite limits so it preserves Abelian group objects. In addition, 


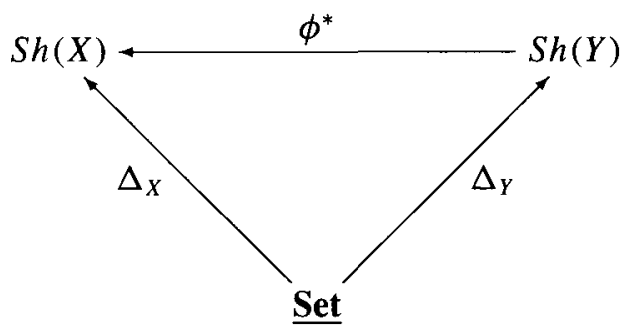

commutes. In particular, $\phi^{*} \Delta_{Y} \mathbf{C}=\phi^{*} \mathbf{C}_{Y, l . c .}=\Delta_{X} \mathbf{C}=\mathbf{C}_{X, l c}$. Thus, $\phi^{*}$ lifts to $\mathrm{C}_{I c}$-modules.

Let $G$ be a $\mathbf{C}_{l c}$-module. A norm on $G$ yields a pseudo-norm, $\|\cdot\|^{*}$, on $\phi^{*} G$ as the transpose of

$$
G(B) \stackrel{\|\cdot\|_{B}}{\longrightarrow} \mathbf{R}_{Y}^{+}(B) \stackrel{-\circ \phi}{\longrightarrow} \mathbf{R}_{X}^{+}\left(\phi^{-1}(B)\right)
$$

or, equivalently, as the composite

$$
\phi^{*} G \stackrel{\phi^{*} \circ\|\cdot\|}{\longrightarrow} \phi^{*} \mathbf{R}_{Y}^{+} \stackrel{t}{\longrightarrow} \mathbf{R}_{X}^{+}
$$

where $\phi^{*} \mathbf{R}_{Y}^{+} \stackrel{t}{\longrightarrow} \mathbf{R}_{X}^{+}$is the transpose of $\mathbf{R}_{Y}^{+} \stackrel{-\circ \phi}{\longrightarrow} \phi_{*} \mathbf{R}_{X}^{+}$. This becomes a norm on $\phi^{\#} G$ after completion. As an example of the techniques used, we prove:

PROPOSITION 5.4. $\|\cdot\|^{*}$ is homogeneous with respect to scalar multiplication by locally constant (complex) functions.

PROOF. Homogeneity for a $\mathrm{C}_{l c}$-module, $G$, means that

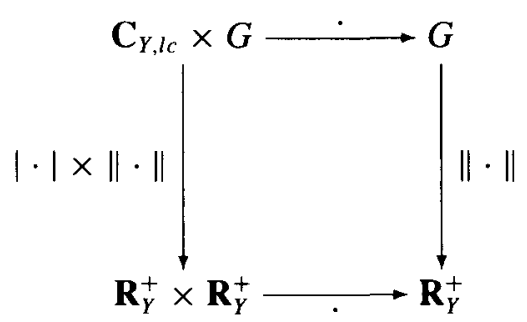

commutes. Apply $\phi^{*}$ to this square and augment to arrive at the following diagram, which we will prove commutative: 




The top square is $\phi^{*}$ of the homogeneity square for $G$, so commutes. To show the bottom square commutes, transpose and use the fact that multiplication is pointwise. The left triangle is the product of two triangles. The second factor commutes by definition of $\|\cdot\|^{*}$. The first factor commutes, since

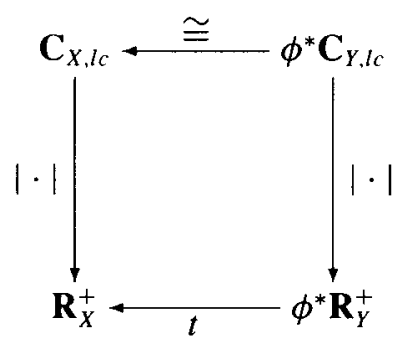

commutes because its transpose does.

Now, complete to get $\phi^{\#}$. For this, we need:

PROPOSITION 5.5. Let $G$ be normed with $\mathbf{C}_{l c}$-homogeneity and suppose it is complete in this norm. Then $\mathbf{G}$ can be made into a normed $\mathbf{C}$-module with $\mathbf{C}$-homogeneity and it is complete.

Proof. For $\alpha(y) \in \mathbf{C}$, let $\alpha_{n}(y) \rightarrow \alpha(y)$ with $\alpha_{n}$ locally constant. Put $(\alpha(y)) \cdot g:=$ $\lim _{n \rightarrow \infty}\left(\alpha_{n}(y)\right) \cdot g$ (the sequence is Cauchy in $G$ ). 
For each $\phi, \phi^{\#}$, as a triple composite, is functorial. Moreover,

PROPOSITION 5.6.

$\underline{\mathbf{M 0 R}}^{o p} \stackrel{()^{*}}{\longrightarrow} \underline{\mathbf{C A T}}$ is pseudo-functorial.

PROOF. $1^{\#} \cong 1$ is straightforward. We will show $(\psi \phi)^{\#} \cong \phi^{\#} \psi^{\#}$ where $\phi$ and $\psi$ are is in the diagram:

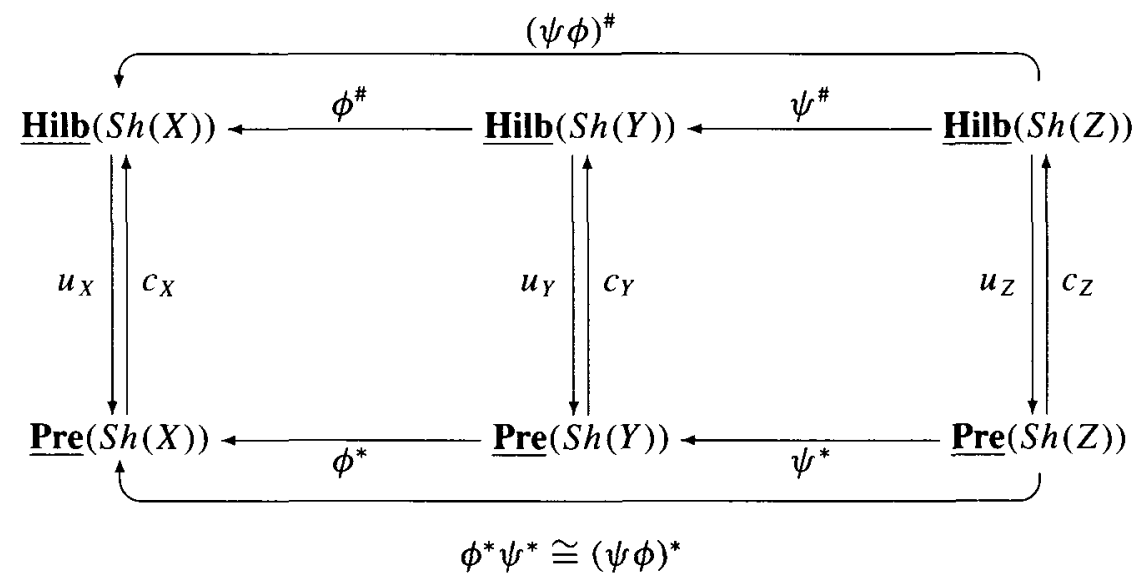

and where $u$ denotes the forgetful functor and $c$ denotes the completion functor.

We first claim that $\phi^{*} c_{Y} \cong c_{X} \phi^{*}$. Let $P \in \underline{\operatorname{Pre}}(\operatorname{Sh}(Y))$. Theorem 4.1 says $P \longrightarrow u_{Y} c_{Y} P$ is a dense inclusion. Then $\phi^{*} P \longrightarrow \phi^{*} u_{Y} c_{Y} P$ is also a dense inclusion since $\phi^{*}$ preserves such. Completing gives $c_{X} \phi^{*} P \cong c_{X} \phi^{*} c_{Y} P$ which is $\phi^{\#} c_{Y}(P)$ by definition. Naturality is easy to check. Similarly, $\psi^{\#} c_{Z} \cong c_{Y} \psi^{\#}$ and $(\psi \phi)^{\#} c_{Z} \cong c_{X}(\psi \phi)^{*}$. Thus, we have

$$
\begin{aligned}
(\psi \phi)^{\#} c_{Z} \cong c_{X}(\psi \phi)^{*} \cong c_{X} \phi^{*} \psi^{*} \\
\cong \phi^{\#} c_{Y} \psi^{*} \cong \phi^{\#} \psi^{\#} c_{Z} .
\end{aligned}
$$

Now, compose on the right with $u_{Z}$ to get $(\psi \phi)^{\#} c_{Z} u_{Z} \cong \phi^{\#} \psi^{\#} c_{Z} u_{Z}$. But $c_{Z} u_{Z} \cong 1$ (Section 4.3). And so, $(\phi \psi)^{\#} \cong \phi^{\#} \psi^{\#}$ as required.

5.3. Direct integration revisited Let $(X, \mathscr{A}, \mu) \stackrel{\left(\phi, \mu_{y}\right)}{\longrightarrow}(Y, \mathscr{B}, \nu)$ be a disintegration and $H \in \underline{\text { Hilb }}(S h(X))$. Put

$$
\left(\int_{\phi}^{\oplus} H\right)(B)=\left\{s \in H\left(\phi^{-1}(B)\right) \mid \int_{\phi^{-1}(y)}\|s\|^{2}(x) d \mu_{y}(x)<\infty \text { almost all } y\right\} .
$$


PROPOSITION 5.7. $\left(\int_{\phi}^{\oplus} H\right)(-)$ is a sheaf.

PROOF. $H\left(\phi^{-1}(-)\right)$ is a sheaf. It is, in fact, $\left(\phi_{*} H\right)(-)$ and the square-integrability condition is on the points, $y$, independent of covers.

We next look at the algebraic properties of $\int_{\phi}^{\oplus}$. Operations $\lceil 0\rceil,-$, and + are as in $H\left(\phi^{-1}(B)\right)$. For example, because $\|\cdot\|_{A}$ is a norm, we have $\left\|s+s^{\prime}\right\|^{2}(x) \leq$ $2^{2}\left(\|s\|^{2}(x)+\left\|s^{\prime}\right\|^{2}(x)\right)$ as in the ordinary sense. If $\beta \in \mathbf{C}(B)$, we can compose with $\phi$ to get $\beta \circ \phi \in \mathbf{C}\left(\phi^{-1}(B)\right)$. For $s \in\left(\int_{\phi}^{\oplus} H\right)(B)$, define scalar multiplication by $\beta \cdot s=(\beta \circ \phi){ }_{\phi^{-1}(B)} s$. Now,

$$
\begin{aligned}
\int_{\phi^{-1}(y)}\|(\beta \circ \phi) \cdot s\|^{2} d \mu_{y}(x) & =\int_{\phi^{-1}(y)}\|\beta \circ \phi(x)\|^{2}\|s\|^{2}(x) d \mu_{y}(x) \\
& =\|\beta(y)\|^{2} \int_{\phi^{-1}(y)}\|s\|^{2}(x) d \mu_{y}(x)<\infty .
\end{aligned}
$$

Furthermore, if $\beta \sim_{Y} \beta^{\prime}$, then $\beta \circ \phi \sim_{X} \beta^{\prime} \circ \phi$ because $\mu\left\{x \mid \beta \circ \phi(x) \neq \beta^{\prime} \circ \phi(x)\right\}=$ $\mu\left(\phi^{-1}\left\{y \mid \beta(y) \neq \beta^{\prime}(y)\right\}\right)=0$, since $\phi \in \underline{\text { MOR }}$.

PROPOSITION 5.8.

$$
\|s\|_{2}^{2}(y)=\left[\int_{\phi^{-1}(y)}\|s\|^{2}(x) d \mu_{y}(x)\right]
$$

defines a norm on $\left(\int_{\phi}^{\oplus} H\right)(B)$ where $[-]$ denotes equivalence class in $\underline{\mathbf{M b l e}}\left(B, \mathbf{R}^{+}\right) / \sim$.

PROOF. As an example, we only prove positive definiteness. Suppose $\int_{\phi^{-1}(y)}\|s\|^{2}(x)$ $d \mu_{y}(x)=0$. By Proposition 2.2, $\int_{X}\|s\|^{2}(x) d \mu(x)=\int_{Y} \int_{\phi^{-1}(y)}\|s\|^{2}(x) d \mu_{y}(x) d v(y)$ $=0$ which implies $s=0$.

REMARKS. (1) Although generally we have left the 'almost all' caveat to the reader to avoid unnecessary repetition, we stress that the relative direct integral consists of local sections which are almost everywhere square-integrable. This is motivated by statements from measure theory, as in the above proof, like: $\int f(x) d x<\infty$ implies $f(x)<\infty$ almost everywhere and not everywhere.

(2) Again, in $S h(X)$, we must choose a representative of the equivalence class $\|s\|$. It is easy to show that ' $\mu$-almost everywhere equality' leads to ' $\mu_{y}$-almost everywhere equality' on almost all of the fibres.

(3) Completeness of the norm of the proposition seems to be difficult to prove in general (however, the examples below are complete). Finding a subsequence such 
that $\sum_{n=1}^{\infty}\left\|s_{n+1}-s_{n}\right\|_{2}(y)$ is finite for almost all $y$, a step crucial to theorem 5.1, is not easy. We avoid the issue as to whether $\int_{\phi}^{\oplus}$ is complete for general $\phi$ by defining new $\int_{\phi}^{\oplus}=c\left(\right.$ old $\left.\int_{\phi}^{\oplus}\right)$ where $c$ denotes completion of a pre-Hilbert sheaf.

We next give some basic examples of relative direct integrals.

EXAMPLE 1. Identity: In this case,

$$
\left(\int_{1}^{\oplus} H\right)(A)=\left\{s \in H(A) \mid \int_{[x]}\|s\|^{2}(t) d l_{x}(t)<\infty\right\}=H(A) .
$$

The finiteness condition on the integral holds for any $s$ since norms are real-valued.

EXAMPLE 2. Terminal Object: For $(X, \mathscr{A}, \mu) \stackrel{(!, \mu)}{\longrightarrow}(1,2$, counting),

$$
\left(\int_{!}^{\oplus} H\right)(B)=\left\{s \in H\left(!^{-1}(B)\right) \mid \int_{!^{-1}(*)}\|s\|^{2} d \mu(x)<\infty\right\} .
$$

If $B=\{*\}$, this is the ordinary direct integral as described above.

EXAMPLE 3. Finite Sets: If $X=\left(n, 2^{n}\right.$, counting), $S h(X) \simeq$ Set $^{n}$. Here, $2^{n}$ is equipped with the 'topology of unions' and a set is covered by a family if the union of the family equals the set. In this case, every set is covered by the collection of its points. An $H \in \underline{\text { Hilb }}(S h(X))$ corresponds to $\left(H_{1}, H_{2}, \ldots, H_{n}\right) \in \underline{\operatorname{Hilb}}\left(\operatorname{Set}{ }^{n}\right)$ and $H(A)=\prod_{x \in A} H(x)$. In particular, $\mathbf{C}(A)=\prod_{x \in A} \mathbf{C}$ and the norm is $H(A) \longrightarrow$ $\mathbf{R}(A),\left\langle h_{x}\right\rangle_{x \in A} \longmapsto\left\langle\left\|h_{x}\right\|\right\rangle_{x \in A}$. Now, suppose, $n \stackrel{\phi}{\longrightarrow} m$ is a disintegration (such is just a function from $n$ to $m$; see [7]). In this case, $\left(\int_{\phi}^{\oplus} H\right)(B)=H\left(\phi^{-1}(B)\right)=$ $\prod_{\left.x \in \phi^{-1}(B)\right)} H(x)$. Operations are pointwise and the norm is the Euclidean one:

$$
\prod_{x \in \phi^{-1}(y)} H(x) \longrightarrow \mathbf{R}, \quad\left\langle h_{x}\right\rangle \longmapsto \sqrt{\sum_{x \in \phi^{-1}(y)}\left\|h_{x}\right\|^{2}}
$$

PROPOSITION 5.9. $\underline{\mathbf{U H i l b}}(S h(X)) \stackrel{\int_{\phi}^{\oplus}}{\longrightarrow} \underline{\mathbf{U H i l b}}(S h(Y))$ is a functor for each $\phi$.

Proof. Suppose $H \stackrel{r}{\longrightarrow} H^{\prime} \in \underline{\mathbf{U H i l b}}(\operatorname{Sh}(X))$. For $s \in\left(\int_{\phi}^{\oplus} H\right)(B)$, we have $\int_{\phi^{-1}(y)}\|\tau s\|^{2}(x) d \mu_{y}(x) \leq b^{2} \int_{\phi^{-1}(y)}\|s\|^{2}(x) d \mu_{y}(x)<\infty$ for almost all $y$.

PROPOSITION 5.10. $\int_{-}^{\oplus}: \underline{\text { Disint }}^{o p} \rightarrow \underline{\text { CAT }}$ is a pseudo-functor. 
ProOF. Example 1 above shows that $\int_{1}^{\oplus}=1$. Let

$$
(X, \mathscr{A}, \mu) \stackrel{\left(\phi, \mu_{3}\right)}{\longrightarrow}(Y, \mathscr{B}, \nu) \stackrel{\left(\psi, v_{3}\right)}{\longrightarrow}(Z, \mathscr{C}, \rho)
$$

be two disintegrations and let $\left(\psi \phi, \theta_{z}\right)$ denote their composition. For $H$ a Hilbert sheaf, the two relevant direct integrals are:

$$
\begin{aligned}
& \left(\int_{\psi \phi}^{\oplus} H\right)(C)=\left\{s \in H\left(\phi^{-1} \psi^{-1}(C)\right) \mid \int_{\phi^{-1} \psi^{-1}(z)}\|s\|^{2}(x) d \theta_{z}(x)<\infty \text { a.a. } z\right\} \quad \text { and } \\
& \left(\int_{\psi}^{\oplus} \int_{\phi}^{\oplus} H\right)(C)=\left\{t \in\left(\int_{\phi}^{\oplus} H\right)\left(\psi^{-1}(C)\right) \mid \int_{\psi^{-1}(z)}\|t\|^{2}(y) d v_{z}(y)<\infty \text { a.a. } z\right\} \\
& =\left\{t \in H\left(\phi^{-1} \psi^{-1}(C)\right) \mid \int_{\phi^{-1}(y)}\|t\|^{2}(x) d \mu_{y}(x)<\infty \text { a.a. } y\right. \text { and } \\
& \left.\int_{\psi^{-1}(z)} \int_{\phi^{-1}(y)}\|t\|^{2}(x) d \mu_{y}(x) d v_{z}(y)<\infty \text { a.a. } z\right\} .
\end{aligned}
$$

The two choices of representatives are 'absorbed' into one choice. We claim that these two sets are equal. We have $\supseteq$ since, by an argument similar to Proposition 2.2, $\int_{\psi^{-1}(z)} \int_{\phi^{-1}(y)}=\int_{\phi^{-1} \psi^{-1}(z)}$. For $\subseteq$, there is a choice of representative of $\|s\|$ to make $\int_{\phi^{-1} \psi^{-1}(z)}\|s\|^{2}(x) d \theta_{z}(x)$ finite for all $z$. Thus, $\int_{\psi^{-1}(z)} \int_{\phi^{-1}(y)}\|s\|^{2}(x) d \mu_{y}(x) d \nu_{z}(y)$ is finite for all $z$ which implies the inside integral is finite for almost all $y$. We have already noted that if the integral is finite for some choice, then it is finite for any choice.

REMARK. We have actually shown that relative direct integration is functorial in $\phi$. In the context of this paper, we are only interested in the fact that it is pseudo-functorial.

We close with some remarks about interesting open problems:

\section{Epilogue}

(1) M0R, as base category for substitution, and Disint, as base category for direct integration, do not have products so this is not classical indexed category theory (in the sense of [4]). The direct integral is a useful construction, however, and perhaps this points to the existence of a generalization of their theory (that is, where the base is not necessarily finitely complete). The power of indexed category theory is in its descriptions of internal completeness and internal category objects. Of course, this is where the finite limits for the base category are required. It would be interesting to explore similar examples and see what fragment of the classical theory remains. 
(2) As we noted in the introduction, one could change the base category and use Grothendieck toposes or 'expand' M0R (or Disint) to a finitely complete category. In these cases, it would be interesting to see what fragment of classical direct integration remains.

(3) It would be interesting to determine what a disintegration yields in $\phi^{*} \dashv \phi_{*}$ for sheaves. It may equip $S h(X)$, regarded as a topos over $S h(Y)$, with a notion of measure. This is not straightforward, however, since passing to sheaf topoi does not capture the measure exactly. A more general notion of 'disintegration' would be required. But, this would be a fruitful enterprise. For example, $\int_{\phi}^{\oplus}$ would simply be the internalization (in $S h(Y)$ ) of $\int^{\oplus}$ (in $\underline{\text { Set)}}$ ).

(4) The direct integral functor is not left adjoint to $\Delta$. For $H$ a Hilbert sheaf and $K$ a Hilbert space, we would require a bijection

$$
\underline{\text { Hilb }}\left(\int^{\oplus} H, K\right) \cong \underline{\text { Hilb }}^{X}(H(-), \Delta(K)) .
$$

As a special case, let $K=\mathbf{C}$ and $H=\Delta(\mathbf{C})=\mathbf{C}_{X}$ so

$$
\underline{\operatorname{Hilb}}\left(L^{2}(X, \mathbf{C}), \mathbf{C}\right) \cong \underline{\operatorname{Hilb}}^{X}(\operatorname{Mble}(-, \mathbf{C}) / \sim, \operatorname{Mble}(-, \mathbf{C}) / \sim) .
$$

Now suppose $(X, \mathscr{A}, \mu)$ is $\left(\mathbf{N}, 2^{\mathrm{N}}, \mu\right)$ with $\sum_{x=1}^{\infty} \mu(x)<\infty$ (to make it a finite measure). The left hom set is $\underline{H i l b}\left(L^{2}(X), \mathbf{C}\right) \cong L^{2}(X)$ (that is, the dual space). An element of the right is a natural transformation

$$
\left\langle\operatorname{Mble}(A, \mathbf{C}) / \sim \stackrel{\psi_{A}}{\longrightarrow} \operatorname{Mble}(A, \mathbf{C}) / \sim\right\rangle_{A \in \mathscr{A}} .
$$

By the sheaf property, such is uniquely determined by singletons: $A=\{x\}$. Now, Mble $(\{x\}, \mathbf{C}) / \sim \cong \mathbf{C}$, boundedness in the $\underline{\mathbf{H i l b}}^{X}$ sense places no condition on the $\psi_{\{x\}}$ 's, and linearity means they are 'ordinary' linear. So $\psi_{\{x\}}$ is just a $1 \times 1$ matrix C $\stackrel{a_{x}}{\longrightarrow}$ C. Thus, each natural transformation, $\psi$, corresponds to a sequence $\left(a_{x}\right)_{x \in N}$. And so, the right hom set consists of all sequences which is much larger than the left. (5) We can, however, end on a more positive note than 4 . First of all, we note that the ' $L$-direct integral' of Banach spaces (defined in an obvious way) works in a context similar to the above counterexample. Specifically, it is well-known that $l^{1}$ is left adjoint to the unit ball functor for Banach spaces and contractions. In future work, we will explore measure-indexed families of Banach spaces, $C^{*}$-algebras, etcetera. These should work better than Hilbert spaces.

For Hilb, we do not have an adjunction but we almost do in the following sense. Let $X$ be as in Remark 4. If we use transformations with uniformly bounded sup norms throughout our theory, the right side of the intended adjunction becomes $L^{\infty}(X)$ for that example. A collection of maps, $H(x) \stackrel{T(x)}{\longrightarrow} K$, whose sup norms are uniformly 
bounded (in $x$ ), yields a unique extension

$$
\int^{\oplus} H(x) d \mu(x) \stackrel{T}{\longrightarrow} K ; s(x) \longmapsto \sum_{x \in X} T(x) s(x) .
$$

This does not give the adjunction since composing a bounded linear transformation with the inclusions, $H(X) \rightarrow \int^{\oplus} H(x) d \mu(x)$, does not give a collection of maps whose sup norms are uniformly bounded. But, $L^{\infty}(X)$ is dense (in the topology of) $L^{2}(X)$ so everything works except at the 'last stage', that of taking limits. This is an interesting phenomenon worth exploring in more detail. For example, one may then explore this 'almost adjunction' in its full generality (with $\int_{\phi}^{\oplus}$ and $\phi^{*}$ ). It is useful to begin with the special case above, however, for a more basic understanding.

\section{References}

[1] J. Dixmier, Les algèbres d'opérateurs dans l'espace Hilbertien (Gauthier-Villars, Paris, 1969).

[2] C. R. Howlett, Universal algebra in topoi (Ph. D. Thesis, McMaster, 1973).

[3] C. Mulvey, Intuitionistic algebra and representations of rings, Mem. Amer. Math. Soc. 148 (1974), 3-57.

[4] R. Paré, D. Schumacher, Abstract families and the adjoint functor theorem, in: Lecture Notes in Math. 661 (Springer, New York, 1978), 1-125.

[5] P. T. Johnstone, Topos theory, London Math. Soc. Monographs 10 (Academic Press, London, 1977).

[6] C. Rousseau, 'Topos theory and complex analysis', in: Lecture Notes in Math. 753 (Springer, New York, 1979), 623-659.

[7] M. Wendt, 'The category of disintegrations,' Cahiers Topologie Géom. Diffèrentielle Catégoriques 35 (1994), 291-308.

Department of Mathematics, Statistics and Computer Science

Dalhousie University

Halifax NS

B3H 4H6

Canada 